\title{
The Cold Stability of Microtubules Increases During Axonal Maturation
}

\author{
Danny F. Watson, ${ }^{1 . a}$ Paul N. Hoffman, ${ }^{1,2}$ and John W. Griffin ${ }^{1}$ \\ Departments of 'Neurology and ${ }^{2}$ Ophthalmology, Johns Hopkins University School of Medicine, Baltimore, Maryland \\ 21205
}

\begin{abstract}
The development of cold-stable microtubules (MTs) was studied in maturing rat sciatic nerves. From 4 to 10 weeks of age, there was a large increase in the proportion of tubulin associated with stable MTs. A greater fraction of axonal tubulin than nonaxonal tubulin was associated with stable MTs. The labeled tubulin remaining behind the peak of slow axonal transport was more highly associated with stable MTs than tubulin in the peak itself. Immunoassay confirmed that the sciatic nerve contains a pool of stable MTs not identical to the peak of tubulin transport. The developmental increase in MT stability is not associated with any increase in the acetylation of tubulin or with alterations in the major MTassociated proteins. One aspect of maturation of the axonal cytoskeleton may involve deposition of tubulin into stable MTs that are either stationary or moving slowly with regard to the peak of transported tubulin.
\end{abstract}

Microtubules (MTs) are assembled from dimeric tubulin subunits. The reverse process, disassembly of MTs into soluble tubulin, occurs normally in living cells (Schulze and Kirschner, 1986, 1987; Sammak and Borisy, 1988; Lim et al., 1989) and can be promoted by cold, a variety of toxins, and increased intracellular calcium. Many cells contain populations of stable MTs that are resistant to depolymerization (Behnke and Forer, 1967; Pipeleers et al., 1977b; Schulze and Kirschner, 1987). Several different mechanisms may contribute to the physical stability of the MTs that resist depolymerization: the presence of special isotypes of tubulin (Brady et al., 1984; Detrich et al., 1987; Binet and Meininger, 1988), acetylation and detyrosination of tubulin (Piperno and Fuller, 1985; Black and Keyser, 1987; Cambray-Deakin and Burgoyne, 1987; Gundersen et al., 1987; Kreis, 1987; Piperno et al., 1987; Schulze et al., 1987; Bulinski et al., 1988; Sale et al., 1988; Black et al., 1989), and the abundance and post translational modifications of MT-associated proteins (MAPs; Hesketh, 1984; Yamamoto et al., 1985; Job et al., 1987; Ohta et al., 1987; Hoshi et al., 1988).

\footnotetext{
Received Dec. 8, 1989; revised May 16, 1990; accepted May 30, 1990.

Aspects of this work were supported by NIH grants NS14784, NS22849, and NS20164. D.F.W. is the recipient of NIH Teacher-Investigator Award NS00983. P.N.H. is the recipient of NIH Research Career Development Award NS00896. We thank Kenneth Fittro and Jay Geyer for their excellent technical assistance. Dr. Diane Griffin provided valuable advice regarding ELISA techniques.

Correspondence should be addressed to Dr. Danny F. Watson, Department of Neurology, Wayne State University, 6E University Health Center, 4201 St. Antoine, Detroit, MI 48201.

a Present address: Department of Neurology, Wayne State University School of Medicine, Detroit, MI 48201

Copyright @ 1990 Society for Neuroscience $0270-6474 / 90 / 103344-09 \$ 03.00 / 0$
}

Mature peripheral nerve axons contain a large population of highly stabilized MTs (Brady et al., 1984; Donoso, 1986; Sahenk and Brady, 1987; Filliatreau et al., 1988; Denoulet et al., 1989). The molecular basis for this stability in axons remains poorly understood; however, proper regulation of the stability of MTs is clearly important for normal maintenance of the axon. For example, drugs such as colchicine or vincristine that reduce MT stability can cause symptomatic neuropathy in humans, marked by distal axonal degeneration (Gottschalk et al., 1968; McLeod and Penny, 1969; Bradley et al., 1970; Riggs et al., 1986; Kuncl et al., 1987).

\section{Relationship of MT stability to axonal maturation}

We have investigated the physical stability of intraaxonal MTs in the rat sciatic nerve 3 to 14 weeks of age and particularly in sensory axons coursing peripherally from the L5 dorsal root ganglion. Before 3 weeks of age, the relative abundance of mRNAs for genes encoding distinct isotypes of beta-tubulin changes to the adult pattern in these sensory neurons (Hoffman and Cleveland, 1988). The expanding myelinated axons during this stage of development rapidly accumulate intraaxonal neurofilaments, and there is an associated slowing of transport of neurofilaments and MTs (Komiya, 1980; Hoffman et al., 1983, 1985). It has not been established whether axons develop a large population of stable MTs prior to or in concert with these other aspects of cytoskclctal maturation. Neither has it been established whether the acetylation of tubulin in maturing axons increases in a fashion similar to early development of neurites of cultured cells (Black and Keyser, 1987). The possibility of a change in the types and relative abundance of MAPs is also a hypothetical mechanism for maturation of the axonal cytoskeleton, but information on this point has been lacking.

Our results indicate that there is a large increase in the population of axonal MTs that resist cold depolymerization from 4 to 10 weeks of age. The data further suggest that this is accomplished in part by deposition of transported tubulin into stable MTs that either are stationary or are transported much more slowly than the bulk of slow axonal transport. There is an unexpected decrease in the abundance of acetylated tubulin during maturation. The MAPs in peripheral nerves differ from the MAPs of the brain but do not show any significant maturational change in type or relative abundance from 3 weeks to 3 months of age.

\section{Materials and Methods}

Labeling of axonal transport. Experimental procedures were performed in male Sprague-Dawley rats under chloral hydrate anesthesia. Proteins were labeled by microinjection of $100 \mu \mathrm{Ci}$ of ${ }^{35} \mathrm{~S}$-methionine dissolved 
in $2 \mu \mathrm{l}$ PBS into the L5 dorsal root ganglion via a glass micropipette. For most groups of animals, the interval between injection and death was chosen so that the peak of labeled transported tubulin would be contained in the nerve segment $20-30 \mathrm{~mm}$ distal to the ganglion, as illustrated in the transport profile in Figure 1. Because axonal transport slows with maturation, different time intervals were allowed at different ages for labeling: animals labeled at 3 weeks of age were killed $7 \mathrm{~d}$ later (4 weeks of age), animals labeled at 6 weeks of age were killed $9 \mathrm{~d}$ later (approximately 7 weeks of age), animals labeled at $7 \frac{1 / 2}{\text { or }} 8^{1 / 2}$ weeks of age were killed $10 \mathrm{~d}$ later ( 9 and 10 weeks of age), and animals labeled at 12 weeks of age were killed $12 \mathrm{~d}$ later (14 weeks of age). Another series of animals, labeled at 3 weeks of age, was studied after longer transport intervals ( 20 or $40 \mathrm{~d}$ after labeling) when the bulk of labeled tubulin had passed beyond the segment $20-30 \mathrm{~mm}$ from the ganglion.

Preparation of tubulin fractions. Previously reported techniques (Pipeleers et al., 1977a; Olmsted, 1981) were adapted to small nerve segments. At $4,7,10$, and 14 weeks of age, the sciatic nerve was exposed, and the nerve segment from 20 to $30 \mathrm{~mm}$ distal to the L5 dorsal root ganglion was excised. The nerve segment was immediately ground with a glass pestle for $30-45 \mathrm{sec}$ in $0.75 \mathrm{ml}$ of an MT-stabilizing solution: $50 \%$ glycerol, $5 \%$ dimethylsulfoxide (DMSO), $0.1 \mathrm{~m}$ piperazine $\mathrm{N}, \mathrm{N}^{\prime}$-ethylsulfonic acid (PIPES) buffer (pH, 6.9), $1.0 \mathrm{~mm}$ EGTA, and $1.0 \mathrm{~mm}$ $\mathrm{MgCl}_{2}$. The homogenate was then centrifuged at $425,000 \times \mathrm{g}$ for 15 $\min$ at $25^{\circ} \mathrm{C}$. The supernatant contained tubulin subunits that were not assembled at the time of homogenization. The pellet, containing both stable and cold-labile MTs was resuspended in $0.75 \mathrm{ml} 0.1 \mathrm{M}$ PIPES buffer ( $\mathrm{pH}, 6.9$ ) containing EGTA and $\mathrm{MgCl}_{2}$ but no DMSO or glycerol. The resuspended pellet was held for $1 \mathrm{hr}$ on ice, then centrifuged as before. At this stage, the supernatant contained soluble tubulin released from cold-labile MTs and the pellet contained the stable MT fraction. The pellet was then dissolved in $8 \mathrm{M}$ urea and 5\% 2-mercaptoethanol in PBS.

Nonaxonal tubulin. In a different group of animals at 14 weeks of age, nonaxonal tubulin of the segment of sciatic nerve $20-30 \mathrm{~mm}$ from the $\mathrm{L} 5$ ganglion was labeled by endoneurial injection of ${ }^{35} \mathrm{~S}$-methionine ( $50 \mu \mathrm{Ci}$ in $1 \mu \mathrm{l}$ injected over $5 \mathrm{~min}$ via a glass micropipette). These experiments took advantage of the fact that mammalian axons lack ribosomes and are incapable of substantial protein synthesis; hence, local labeling preferentially labels proteins of Schwann cells and other nonaxonal elements in nerves. Nerves were subjected to the standard MT fractionation technique $2 \mathrm{hr}$ after labeling; preliminary experiments indicated that much less labeled tubulin could be recovered by $18 \mathrm{hr}$ after labeling.

Determination of tubulin content of fractions. Two different assays of the tubulin content of the nerve fractions were performed on aliquots of the same fractions. The content of labeled transported tubulin was determined by SDS-PAGE, then by cutting the tubulin bands from the gel, digesting them in hydrogen peroxide, and determining ${ }^{35} \mathrm{~S}$ content by scintillation counting. Twice-cycled rat brain tubulin was electrophoresed on adjacent lanes to permit identification of the tubulin band. Nonaxonal tubulin, labeled by local injection into the nerve, was analyzed in the same fashion as axonal tubulin. In some additional experiments, locally labeled tubulin or axonally transported labeled tubulin was subjected to a preliminary immunoprecipitation with an excess of polyclonal rabbit anti-tubulin serum (Sigma) bound to Affi-Gel beads (BioRad). The beads were repeatedly washed with PBS, then the bound tubulin was eluted with SDS, urea, and 2-mercaptoethanol and electrophoresed, and the ${ }^{35} \mathrm{~S}$ content of the tubulin band was counted.

For all experiments with labeled tubulin, the total tubulin $\mathrm{cpm}$ was computed for each individual nerve as the sum of the cpm recovered from the tubulin band for all 3 fractions of that nerve. The cpm in the tubulin band from each fraction was then expressed as a percentage of the total tubulin $\mathrm{cpm}$ in that individual nerve. Means and SEs were then calculated from these percentages for $n=8$ nerves per group.

The total tubulin content of each fraction was independently determined by an enzyme-linked immunosorbent assay in microtiter wells. Wells were treated for $30 \mathrm{~min}$ with a $0.1 \%$ solution of polylysine, then washed extensively $0.1 \%$ TWEEN-20 in PBS after this step and each subsequent step. Aliquots of the nerve fractions were diluted 10- to 200fold in $0.8 \mathrm{~m}$ urea with $0.5 \% 2$-mercaptoethanol in PBS, and nerve proteins were allowed to adsorb to the plate overnight at $4^{\circ} \mathrm{C}$. Excess protein-binding sites were blocked with $3 \%$ horse serum for $1 \mathrm{hr}$. Primary alpha-tubulin and beta-tubulin monoclonal antibodies, obtained from Amersham as ascites fluid, were diluted 1:2000 in PBS before use. Wells were exposed to primary antibody for $2.5 \mathrm{hr}$ followed by biotiny-

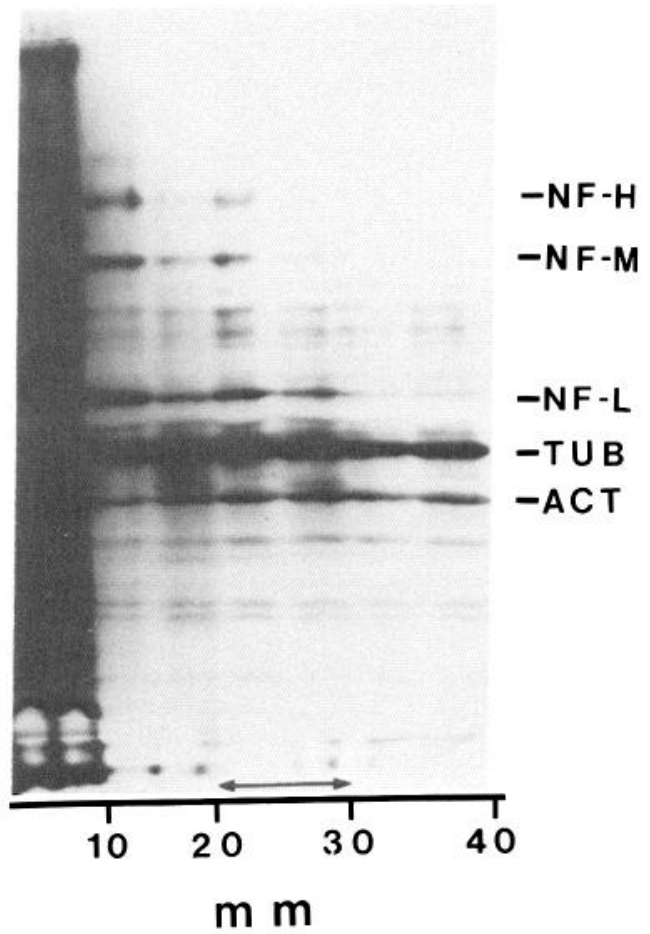

Figure 1. Profile of axonal transport $7 \mathrm{~d}$ after labeling the L5 ganglion in a 3-week-old rat. Gel electrophoresis of 5-mm nerve segments was performed with fluorographic detection of labeled proteins. Tubulin labeling $(T U B)$ is most intense in the segments $20-30 \mathrm{~mm}$ from the ganglion; these segments were used in subsequent experiments on MT stability (arrow). The triplet neurofilament proteins $(N F-H,-M$, and $-L$ ) have just reached the segment $20-25 \mathrm{~mm}$ from the ganglion; actin $(A C T)$ shows a distribution similar to tubulin.

lated equine anti-mouse IgG and subsequently with avidin-biotin-peroxidase complexes (Vector Labs). The amount of bound peroxidase was detected by enzymatic reaction with hydrogen peroxide and $o$-phenylene-diamine in citrate buffer $(\mathrm{pH}, 5.0)$. The reaction was stopped by addition of $5 \mathrm{~N} \mathrm{H}_{2} \mathrm{SO}_{4}$. Optical density was determined at $492 \mathrm{~nm}$ with an automatic Titertek ELISA reader.

Duplicate wells were prepared for each sample for each antibody. Rat brain tubulin prepared by 2 cycles of polymerization was used as a standard; concentrations of $0,25,50$, and $100 \mathrm{ng} / \mathrm{ml}$ tubulin monomer were run in parallel with experimental samples. Before final determination of tubulin content, preliminary immunoassay on serial dilutions of nerve samples was performed to identify dilutions which corresponded to $25-50 \mathrm{ng} / \mathrm{ml}$ and to verify that optical density was within a linear range for serial dilution of nerve. Addition of tubulin as an internal standard demonstrated that the presence of nerve homogenate caused no interference with the detection of tubulin (Fig. 2).

No significant differences between alpha- and beta-tubulin content were identified for any group of samples. Therefore, in subsequent data analysis, the sum of alpha- and beta-tubulin for each individual sample was used; the SE for each group was less for this sum than for either monomer considered alone.

Immunoblots. Segments $(10 \mathrm{~mm})$ of sciatic nerve were removed from 3- or 12-week-old rats ( $n=6$ rats of both ages) and homogenized in $400 \mu 18 \mathrm{M}$ urea with 5\% 2-mercaptoethanol in PBS. SDS was added to $40-\mu 1$ aliquots to a final concentration of $1 \%$, and the solutions were boiled for $2 \mathrm{~min}$, then centrifuged at $16,000 \times \mathrm{g}$. Pooled aliquots from 2 nerves were loaded on each lane of a 3-10\% polyacrylamide gel. After electrophoresis, the proteins were transferred to nitrocellulose overnight (Towbin et al., 1979). The nitrocellulose was blocked with $0.5 \%$ milk in PBS, then exposed to 1 of the following antibodies: Amersham alphatubulin antibody, antibody 6-11-B to acetylated alpha-tubulin (a gift from Dr. G. Piperno), MAP2-C monoclonal antibody (a gift from Dr. A. Matus), or Tau-2 antibody (a gift from Dr. L. Binder), each at a dilution in ascites fluid of 1:2000. Blots were extensively washed, then 

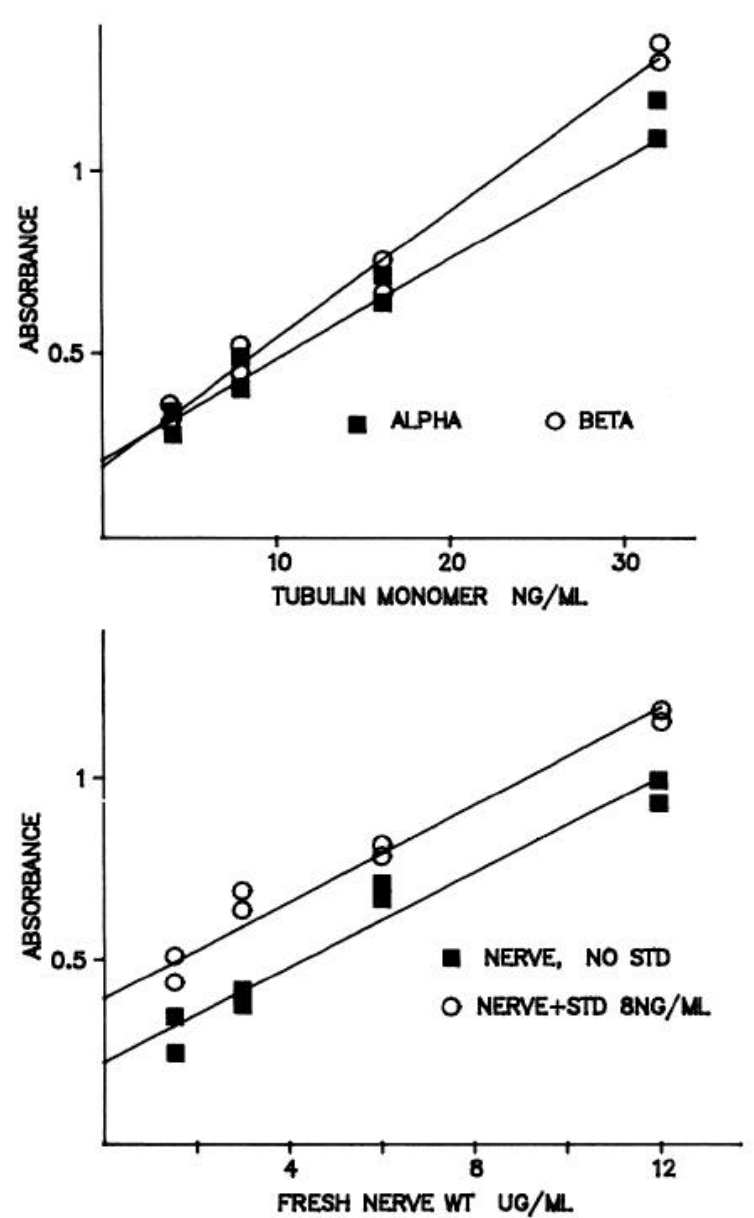

Figure 2. Tubulin immunoassay. Duplicate wells and least-squares regression lines are shown. Top, Absorbance as a function of concentration of purified tubulin standard fit a straight line for alpha- and betatubulin monoclonal antibodies. Bottom, Dilutions of nerve homogenates gave a linear relationship of optical density to concentration (solid squares). Addition of a known concentration of purified tubulin standard (STD) to the nerve homogenates (open circles) showed strictly additive effects, that is, no interference of nerve homogenate proteins with detection of tubulin. Data illustrated are for the beta-tubulin antibody.

the bound primary antibody was detected with goat anti-mouse secondary antibody and peroxidase-antiperoxidase complexes.

Preparation of $M T$ and associated proteins from the PNS. Bilateral sciatic nerves from hip to knee were rapidly removed from 10 rats at 3 weeks of age or 6 rats at 12 weeks of age, frozen at $-70^{\circ} \mathrm{C}$, then thawed to partially disrupt stable MT. Other preparations were made from fresh rat brain. Tissue was homogenized in 1 vol 0.1 m PIPES buffer $(\mathrm{pH}$, 6.9) with $1 \mathrm{~mm}$ EGTA, $1 \mathrm{~mm} \mathrm{MgCl}_{2}, 1 \mathrm{~mm}$ phenylmethylsulfonyl fluoride (PMSF), and $2 \mu \mathrm{g} / \mathrm{ml}$ leupeptin. The remainder of the MT isolation procedure was that of Vallee (1982), including washing with $0.35 \mathrm{M}$ $\mathrm{NaCl}$ in the presence of $20 \mu \mathrm{M}$ taxol to partially separate MAPs from the MT.

\section{Results}

\section{Characterization of tubulin fractions}

Electron micrographs of gultaraldehyde-fixed pellets revealed abundant profiles of MTs in the final stable MT fraction. In some instances, these MTs appeared to be embedded in fragments of axonal cytoskeleton with associated neurofilaments (Fig. 3A). Myelin and the axolemma were disrupted by the procedure. There was a characteristic composition of the fractions apparent on either Coomassie blue staining or on fluo-
A

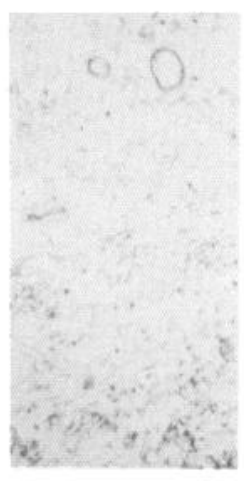

B

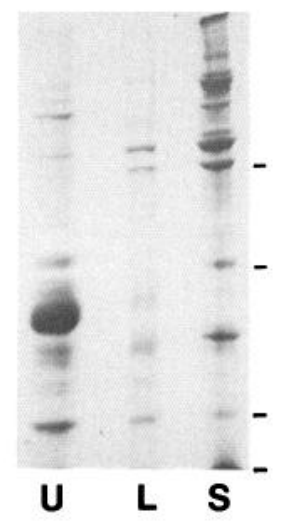

C

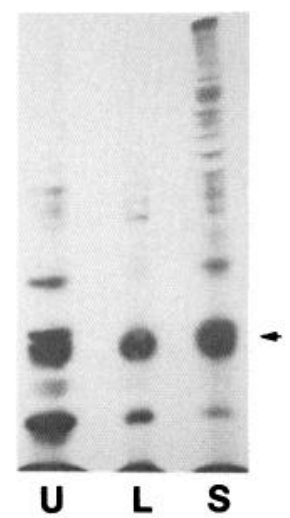

Figure 3. Characterization of tubulin fractions. A, Electron micrograph of the final stable MT pellet. A typical fragment of axonal cytoskeleton is shown. MTs with diameters of $24-25 \mathrm{~nm}$ are abundant; their orientation is more variable than in intact axons. $B$, Coomassie bluestained gel of tubulin fractions from a single nerve in a rat at 4 weeks of age. U, fraction containing unassembled subunits; L, fraction with tubulin from labile MTs; S, fraction with stable MTs. Dashes indicate MW markers: $92,68,45$ and $25 \mathrm{kDa}$. $C$, Fluorogram of the same gel (transport labeled $7 \mathrm{~d}$ before harvest). Arrow indicates the mobility of tubulin standard. A substantial portion of the labeled tubulin appears in each fraction.

rography of gels (Fig. $3 B, C$ ); for example, actin was most abundant in the initial supernatant (fraction with unassembled subunits), and neurofilament proteins were nearly all contained in the final pellet (fraction with stable MT).

\section{Axonal maturation and MT stability}

There was a significant maturational increase in stability of MTs from 4 to 14 weeks of age, especially in the interval of 4-10 weeks of age. Immunoassay of total tubulin contained in the nerve indicated an increase in the absolute amount of tubulin in each fraction; however, a much greater proportion of the tubulin was associated with stable MTs by 10 weeks of age (Fig. 4). The labile MT population by immunoassay was small at each age, and consequently, the increase in the proportion of stable MTs by ELISA was accompanied by a parallel decrease in the proportion of tubulin in the unassembled fraction.

A large maturational increase in the proportion of tubulin associated with stable MTs was also seen when labeled transported tubulin was assayed (Fig. 4), though there was a systematic difference in the proportions with the 2 methods of tubulin quantitation (see below). At 4 and 7 weeks of age, a significant portion of the labeled transported tubulin was recovered with the labile MT fraction (Fig. 4). The absolute amount of radioactive tubulin did not change significantly with age: $15,375 \pm$ $3635 \mathrm{cpm}$ at 4 weeks and $14,155 \pm 1795 \mathrm{cpm}$ at 10 weeks of age.

The proportion of radioactive tubulin in the form of stable and labile MTs was not critically affected by the time allowed for transport within a few days (though very long intervals so that the peak of tubulin transport passed beyond the assayed segment had a considerable effect, as decribed below). For example, for rats labeled at 8 weeks of age, transport intervals of 5 or $10 \mathrm{~d}$ gave indistinguishable results for tubulin fractions: $52.5 \pm 4.6 \%$ and $44.8 \pm 4.8 \%$ of tubulin in the stable MT fractions, respectively ( $n=8,0.2>p>0.1$ by $t$-test). 

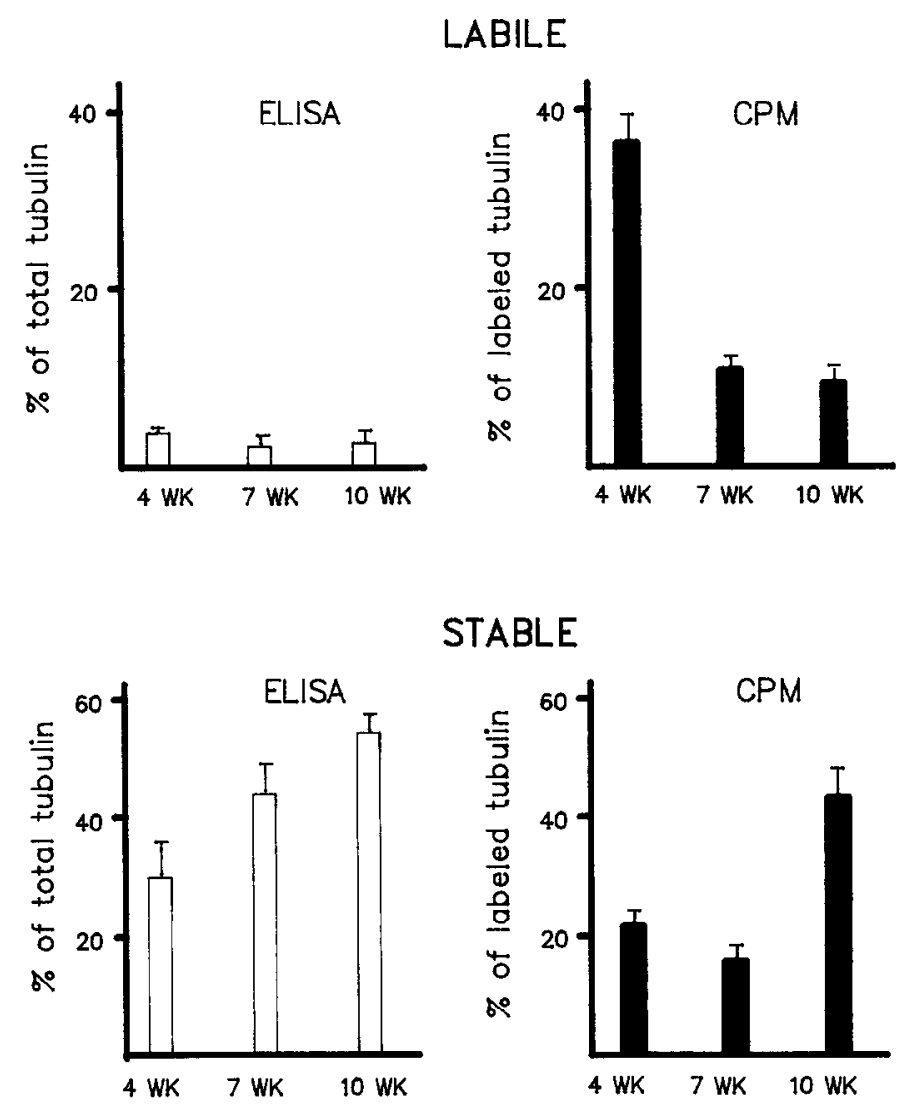

Figure 4. Maturational change in stability properties of MTs. MT fractions werc preparcd from sciatic nerves containing the peak of labeled transport. Tubulin content was measured either by immunoassay (open bars) or by counting ${ }^{35} \mathrm{~S}$ from gel bands (solid bars). Results are expressed as a percentage of the total tubulin recovered in the 3 fractions for immunoassay or as a percentage of the total $\mathrm{cpm}$ recovered in the tubulin bands from the 3 fractions. Bars indicate SE for $n=8$ nerves. $T o p$, Labile MT fraction. There is a maturational decrease in the content of labeled transported tubulin in the labile fraction. At each age, the transported labeled tubulin was more highly associated with labile MTs than the total nerve tubulin by immunoassay. Bottom, Stable MT fraction. There is a maturational increase in the proportion of tubulin associated with stable MTs. At each age, the transported labeled tubulin was less associated with stable MTs than the total tubulin by immunoassay.

\section{Comparison of axonal and nonaxonal tubulin pools}

At each age studied, the proportion of tubulin in the labile MT fraction was smaller by immunoassay than that obtained by analysis of the labeled transported tubulin, and the stable MT fraction was larger by immunoassay. These results suggested that there is a pool of stable MTs in the sciatic nerve that are distinct from the population of labeled MTs in the peak of slow axonal transport. In principle, this $\mathrm{MT}$ pool might be extra- or intraaxonal.

The stability of nonaxonal MTs was assayed by local labeling of the nonaxonal structures of nerves, followed by standard fractionation and gel electrophoresis. Because it was uncertain whether other labeled proteins at the same apparent $\mathrm{MW}$ as tubulin might be prominent in the locally labeled material, a portion of each fraction was subjected to immunoprecipitation of tubulin before electrophoresis. The proportion of locally labeled nonaxonal tubulin associated with stable MTs was much less than the stable MT fraction of transported tubulin (Fig. 5).

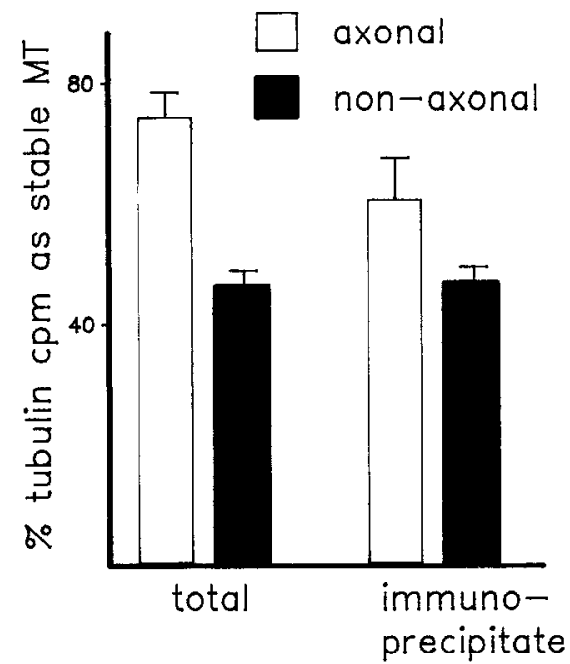

Figure 5. Comparison of axonal versus nonaxonal tubulin. Tubulin fractions from mature nerves ( 14 weeks of age) containing labeled axonal transport were compared to 14-week-old nerves labeled by local endoneurial injection of ${ }^{35} \mathrm{~S}$-methionine. Percentage of the total tubulin cpm in each of the 3 fractions was determined for $n=8$ nerves; the mean \pm SEM of this percentage is shown. Total, Standard gel electrophoresis and counting of the tubulin bands; no immunoprecipitation of fractions prior to electrophoresis. Immunoprecipitation, Tubulin from each fraction was precipitated with rabbit polyclonal anti-tubulin antibody bound to agarose beads prior to electrophoresis. The proportion of axonal stable MTs was greater than the nonaxonal stable MT fraction. There was no significant difference in the percentages with or without immunoprecipitation prior to electrophoresis.

This difference was apparent whether the entire labeled nerve fraction or the immunoprecipitated tubulin cpm from each fraction was analyzed (Figure 5). Hence, the local labeling experiments give no evidence for any large pool of extraaxonal stable MTs to explain the observed differences between the peak of labeled transport and the total tubulin in the nerve, and they suggest that there might be different intraaxonal pools of tubulin.

\section{$M T$ stability and the tubulin remaining behind the peak of slow axonal transport}

The presence of an intraaxonal pool of tubulin that is highly associated with stable MTs was directly demonstrated by experiments that examined the labeled tubulin remaining in the sciatic nerve after the peak of slow axonal transport had passed. Three groups of animals were labeled at 3 weeks of age and allowed to survive for various periods $(7,20$, or $40 \mathrm{~d}$ ) before the nerves were removed and the tubulin fractionated. The association of labeled transported tubulin with stable MTs increased substantially with increasing survival interval after labeling (Fig. 6). Because the transported labeled tubulin gradually moves beyond the assayed segment, less total tubulin radioactivity was recovered at $20 \mathrm{~d}$ and much less at $40 \mathrm{~d}$ after injection: $11,525 \pm 560 \mathrm{cpm}$ (mean $\pm \mathrm{SEM}$ ) and $1595 \pm 175 \mathrm{cpm}$, respectively, versus the peak ( $7 \mathrm{~d}$ ) value of $15,375 \pm 3635 \mathrm{cpm}$.

The high degree of association of residual tubulin with stable MTs was not simply an effect of maturation of the nerve during the transport interval. Animals that were the same age ( 9 weeks) at death but labeled at $7 \frac{1}{2}$ instead of 3 weeks of age, so that the peak of labeled tubulin would be present in the nerve, showed a significantly smaller proportion of tubulin in the stable MT fraction (Fig. 7) compared to the residual tubulin. At 9 weeks 


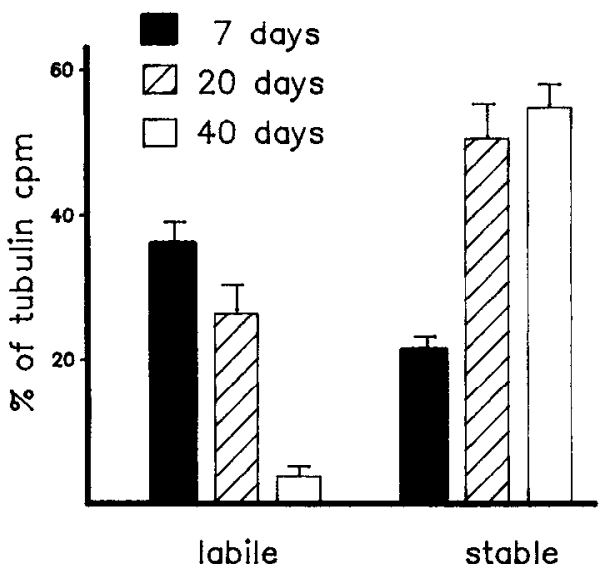

Figure 6. Effect of transport interval on MT stability. Groups of rats labeled at 3 weeks of age were allowed to transport for $7 \mathrm{~d}$ (sufficient for the peak of tubulin to reach the assayed segment) or for 20 or $40 \mathrm{~d}$ (assayed segment contained residual transported tubulin at these times). The proportion of tubulin recovered in the labile MT fraction decreased, and the proportion in the stable MT fraction increased, with greater times after labeling. Mean and SEM for $n=8$ nerves are illustrated.

of age, there was also a greater proportion of the peak of tubulin in association with labile MTs when compared to the residual tubulin (Fig. 7).

\section{Acetylated tubulin immunoreactivity}

The MTs in the stable fraction were enriched in acetylated tubulin immunoreactivity as compared to the labile MTs. Quantitative immunoassay was performed on duplicate sets of wells plated with tubulin fractions from 10 -week-old nerves $(n=8)$ employing either the Amersham alpha-tubulin antibody or the acetylated tubulin antibody 6-11-B. Each antibody was used at saturating concentration, and all secondary steps were carried out in parallel (except that twice as much time was allowed for development of color for the acetylated tubulin wells). Under these conditions, the optical density for the acetylated tubulin in the stable MT fraction was $36.5 \pm 2.1 \%$ of the optical density for the total alpha-tubulin, versus only $6.5 \pm 2.0 \%$ for the labile MT fraction. The "unassembled" fraction gave an intermediate value of $21.7 \pm 3.2 \%$. Expressed another way, there was a 5.6fold enrichment in the acetylated tubulin in the stable MTs as compared to the labile MT fraction, and the stable MT fraction accounted for more than $70 \%$ of the total acetylated tubulin immunoreactivity.

Although the stable MT fraction was enriched in acetylated tubulin immunoreactivity, the developmental increase in MT stability was not mediated by an increase in the abundance of acetylated tubulin. Total immunoreactivity of nerve MTs toward an antibody against acetylated alpha-tubulin showed a decrease from 3 weeks to 3 months of age (Fig. 8). This decrease was apparent with immunoblots or with quantitative immunoassay. The decrease was a modest $29 \%$ when total immunoreactivity per unit length of nerve was considered; however, when the immunoreactivity was adjusted for the greater total tubulin content (or the greater total protein content), the relative abundance of the acetylated epitope at 3 months of age was only $20 \%$ of the value at 3 weeks of age.

Immunoblots revealed that more than 1 band in the region of tubulin reacts with the antibody 6-11-B. The higher apparent

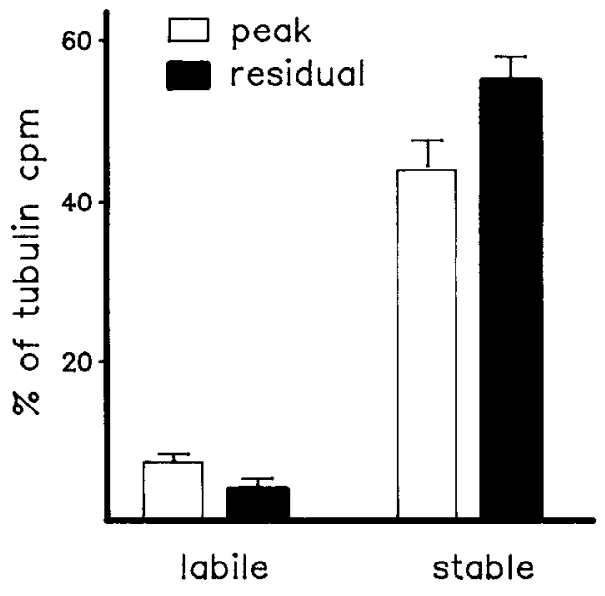

Figure 7. Comparison of the transported peak and residual tubulin in rats of the same age. Nerve segments were harvested at 9 weeks of age from rats labeled $10 \mathrm{~d}$ before (segment containing the peak of tubulin transport) or $40 \mathrm{~d}$ before (containing residual or very slowly transported tubulin; data redrawn from Fig. 6 for comparison). The residual tubulin is more highly associated with stable MTs and less with labile MTs than the peak of transport ( $n=8$ nerves each group; $p<0.05$ by 2 -tailed $t$-test for the labile MT and the stable MT differences).

MW band comigrates with the alpha-tubulin band as identified by the Amersham alpha-tubulin antibody. A second band with lower apparent MW showed the most abundant reaction with the antibody to the acetylated epitope at 3 weeks of age. This band was much less prominent by 3 months of age. A fainter band between these 2 major bands was just visible at either age in heavily loaded lanes.

Although suitable standards for exact quantitation of acetylated alpha-tubulin from the rat are not available, immunoblots suggest that acetylated forms are only a small proportion of the total tubulin. The immunoblots in Figure 8 were performed strictly in parallel with strips of nitrocellulose transferred from the same gel, with the same secondary reagents, and with the primary antibodies at saturating concentrations. The reaction with the acetylated tubulin antibody was much fainter than with the alpha-tubulin antibody, despite the fact that the lanes for the acetylated antibody were loaded with 10 times the total amount of nerve homogenate and developed for 5 times as long at the final enzymatic detection step.

\section{MAPS of peripheral nerve}

Silver- or Coomassie blue-stained gels confirmed that tubulin was the most abundant protein in the taxol-stabilized MTs from peripheral nerves. Of the MAPs released by extraction with 0.35 $\mathrm{M} \mathrm{NaCl}$ (in the presence of $20 \mu \mathrm{M}$ taxol), the most abundant were 2 proteins migrating as broad bands between 60 and 65 $\mathrm{kDa}$, probably tau proteins (Fig. 9). One band at approximately $140 \mathrm{kDa}$ largely remained associated with MTs in the presence of $0.35 \mathrm{M} \mathrm{NaCl}$; the other MAPs, including bands at 120 and $85 \mathrm{kDa}$, were efficiently extracted by this solution. The identity of these bands is not yet confirmed by other techniques; they are "microtubule-associated" in the operational sense of selective pelleting with assembled taxol-stabilized MTs after initial solubilization from the nerve.

These MAPs from nerves differed in relative abundance from MAPs isolated from the brain by the same technique. HighMW MAPs from the sciatic nerve also included 2 closely spaced 


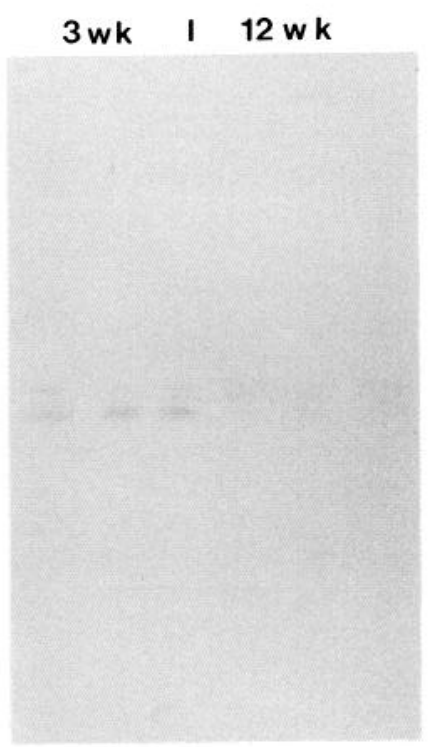

$6-11 B-1$

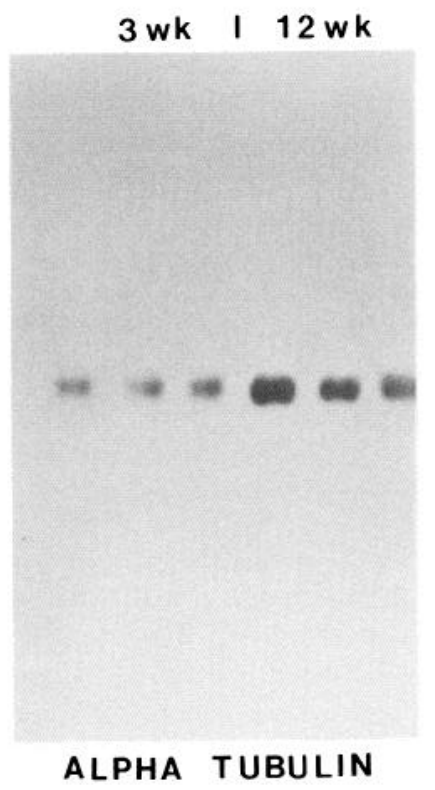

Figure 8. Immunoblots comparing acetylated tubulin (antibody 6-11B) and total alpha-tubulin (antibody from Amersham) at 3 or 12 weeks of age. Each lane was loaded with nerve homogenate in SDS from a different animal. Lanes for 6-11- $B$ were loaded with material from a total of $1 \mathrm{~mm}$ of nerve; those for the alpha-tubulin antibody were loaded with material from a total of $0.1 \mathrm{~mm}$ of nerve. There was more total immunoreactivity toward antibody $6-11-\mathrm{B}$ at 3 weeks of age, despite the fact that there was much less total tubulin. The major band reacting with 6-11-B at 3 weeks of age had a lower apparent MW than the bulk of alpha-tubulin.

bands with mobility similar to MAP2 from the rat brain and reactive with the MAP2-C monoclonal antibody on immunoblots (data not shown). Two bands from sciatic nerves with MWs of approximately $60-65 \mathrm{kDa}$ reacted with the Tau-2 antibody, which reacts with both the nonphosphorylated and the phosphorylated forms of tau (data not shown).

There was no change in the relative amount or the electrophoretic mobility of any of the prominent MAP bands during maturation from 3 weeks to 3 months of age. There was a developmental increase in the abundance of these bands of roughly proportional to the increase in the total tubulin content. No shift in tau or MAP2 electrophoretic mobility by immunoblot was apparent with maturation from 3 to 12 weeks of age.

\section{Discussion}

\section{Stable MT and intraaxonal pools of tubulin}

Between 4 and 10 weeks of age, there is a large increase in the population of cold-stable MTs in the rat sciatic nerve, with a modest further increase from 10 to 14 weeks of age (only labeled transported tubulin was analyzed at 14 weeks). This increase is considerably later than the maturational increase in MT stability in the rat cerebellum (Faivre et al., 1985). The increase in MT stability in the sciatic nerve is apparent with immunoassay of total nerve tubulin or with analysis restricted to labeled axonally transported tubulin. Although both techniques show a maturational increase in MT stability, the results are not identical, even when performed on aliquots of the same solutions.

At each age studied, a greater proportion of tubulin was present as stable MTs by immunoassay as compared to analysis of labeled transported tubulin. This suggests that the nerve as a

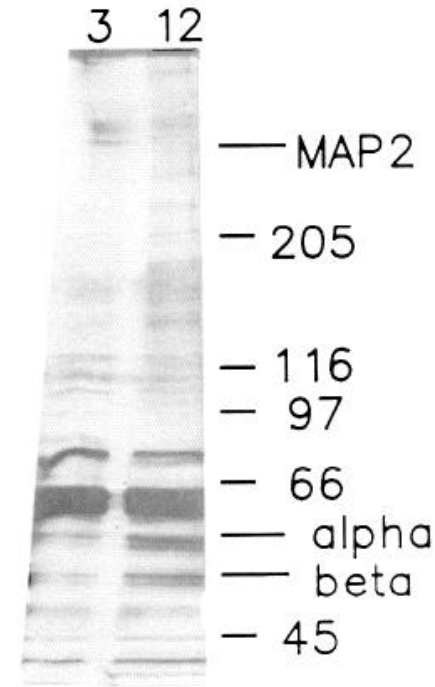

Figure 9. SDS gel of MAPs from sciatic nerve stained with Coomassie blue followed by silver. Taxol-stabilized MTs were prepared from sciatic nerves at 3 weeks (3) or 12 weeks (12) of age. Nerve MTs were then extracted with $0.35 \mathrm{M} \mathrm{NaCl}$ and centrifuged to give soluble MAPs in the supernatant. Positions of MAP2 and alpha- and beta-tubulin (from brain MTs) and of $M W$ markers are indicated. The most prominent MAPs are at $60-65 \mathrm{kDa}$, possibly tau proteins. Other reproducible bands have MW of approximately 140,120 , and $85 \mathrm{kDa}$.

whole contains a larger population of stable MTs than does the peak of slow axonal transport of tubulin. Assay of locally labeled nonaxonal MTs indicates that the tubulin in these nonaxonal structures of nerves is less highly associated with stable MTs than with axonal tubulin, and therefore, nonaxonal sources of tubulin cannot represent the highly stabilized MTs observed by immunoassay. It therefore appears that different intraaxonal pools of tubulin are associated witih MTs of different stability.

The sciatic nerve contains motor axons as well as sensory axons. If a very high proportion of the tubulin in motor axons were in the form of stable MTs, this might account for the difference between immunoassay of total nerve tubulin and the labeled transported tubulin in sensory axons. Filliatreau and colleagues (1988), employing techniques broadly similar to those employed here, found $68-75 \%$ of labeled tubulin in an insoluble MT fraction from motor axons that were 13 weeks old at harvest. This is quite comparable to the $68 \%$ (average of the determinations with and without immunoprecipitation) of labeled tubulin found for the 14-week-old sensory axons in the present study. Hence, it seems unlikely that mature motor axons contain a much larger population of stable MTs than sensory axons. Preliminary determinations in this laboratory of labeled tubulin fractions from L5 motor axons at 10 weeks of age also give values similar to those observed for sensory axons (P. N. Hoffman, unpublished observations).

The solubility of the labeled tubulin that moves ahead of the peak of transported tubulin has also been examined, and a smaller amount of this rapidly moving tubulin was found in association with insoluble polymers (Filliatreau et al., 1988). Therefore, the labeled tubulin moving ahead of the main peak of tubulin transport is not likely to represent a pool of highly stable MTs.

A more direct demonstration of an intraaxonal pool of tubulin highly associated with stable MTs was obtained in this study by analysis of the residual labeled tubulin after the peak of 
labeled transported tubulin had passed. The high degree of association of this residual labeled tubulin with stable MTs suggests that there is deposition of some tubulin into MTs that are stable and are either stationary or moving at a rate much slower than that of the bulk of slow axonal transport. This hypothesis, that axons contain a stationary (or quasi-stationary) population of MTs that slowly exchange with passing transported material, has a parallel in previous observations of stationary (or nearly stationary) labeled neurofilaments within the distal optic nerve axons of the mouse (Nixon and Logvinenko, 1986). The possibility that labeled transported cytoskeletal proteins may exchange with proteins already resident in the axoplasm has also been proposed as a possible mechanism for the slowing of the rate of transport of labeled proteins at increasing distances from the cell bodies of motor axons (Watson et al., 1989).

\section{Relationship of tubulin fractions to tubulin state in vivo}

The fractionation described in this study employs highly artificial conditions, so the correspondence of these fractions to the form of tubulin in vivo is not precise. The technique is likely to underestimate the population of stable MTs. The initial homogenization solution lacks GTP and therefore should not promote fresh polymerization of subunits into MT. Furthermore, there is rapid dilution of the axoplasmic volume by roughly 100-fold during the homogenization, which should further disfavor fresh polymerization. On the other hand, the vigorous homogenization required to disperse peripheral nerves may mechanically disrupt some MTs that would otherwise be stable to cold exposure. Because acetylated tubulin generally occurs in stable MTs (Piperno and Fuller, 1985; Piperno et al., 1987; Bulinski et al., 1988), the presence of some acetylated tubulin immunoreactivity in the "unassembled" tubulin fraction suggests that some stable MTs were disrupted during fractionation. Nonetheless, the 5-fold enrichment of acetylated tubulin in the stable MTs as compared to the labile MT fraction indicates that there is some correlation between the fractions produced in these experiments and the state of the tubulin and MTs in situ. Electron microscopy also confirms that intact MT segments are abundant in the stable MT pellet. It is not known whether this fraction contains insoluble tubulin in the form of oligomers or structures other than MTs.

Another methodologic consideration is the use of 1-dimensional gel electrophoresis to separate labeled tubulin from other proteins. This strategy depends on the simple composition of the labeled material carried by slow axonal transport in peripheral nerves. Prior 2-dimensional gel electrophoresis and peptide mapping of labeled transported material from motor axons have shown that tubulin is indeed the most abundant labeled protein at this electrophoretic mobility (Brady et al., 1984; Tashiro et al., 1984; Tashiro and Komiya, 1989). Two results reported here further indicate that no other labeled polypeptides with the same electrophoretic mobility as tubulin unduly influence our results. First, there is a prominent maturational increase in MT stability by immunoassay, which docs not depcnd on clcctrophoretic separation. Second, the proportion of labeled tubulin in the 3 fractions was the same for lanes loaded with entire fractions or loaded with tubulin immunoprecipitated from the fractions before electrophoresis.

\section{Mechanisms of MT stability}

The present studies investigated 2 molecular mechanisms that might account for increased MT stability with age. The acety- lation of alpha-tubulin, as determined by immunoassay, did not increase; instead, there was an absolute decrease from 3 weeks to 3 months of age. This suggests that acetylation of alphatubulin is not an important event in the stabilization of mature axonal MTs, in contrast to the case of neurites extending from cultured neurons (Black and Keyser, 1987; Black et al., 1989). It is valid to conclude that immunoreactive acetylated tubulin does not increase in parallel with the increase in MT stability, whether only the band at the same mobility as alpha-tubulin is considered, or whether both bands (including the one at lower apparent $\mathrm{MW}$ ) are considered together.

The reaction of the acetylated tubulin antibody with a band below the usual mobility of alpha-tubulin in the 3-week-old nerve was unexpected. Because of the specificity of this antibody for acetylated alpha-tubulin in other systems (Piperno and Fuller, 1985; Piperno et al., 1987; Sale et al., 1988), it seems likely that a posttranslational modification of acetylated alpha-tubulin has led to the altered electrophoretic mobility. One alternative is that limited proteolysis has generated a fragment with slightly lower molecular mass but with preserved immunoreactivity to 6-11-B. This seems less likely because no similar band reacted with the alpha-tubulin monoclonal antibody, even though aliquots of the same nerve sample, electrophoresed on different lanes of the same gel, were used for this experiment. Other alternatives include the presence of a novel genetically determined isoform of alpha-tubulin, cross-reaction of the primary antibody with a protein other than tubulin, or cross-reaction of the secondary antibody used for detection with a nerve component, this cross-reaction becoming apparent only with the longer times for enzymatic reaction employed with the acetylated tubulin antibody.

Changes in abundance, types, or posttranslational modifications of MAPs can affect the stability of MTs (Hesketh, 1984; Yamamoto et al., 1985; Job et al., 1987; Baudier and Cole, 1988; Hoshi et al., 1988), and there are changes in MAPs of the PNS during embryonic development (Tucker et al., 1988). Hypothetically, changes in MAPs during the period of maturation from 3 to 12 weeks of age might exert a major influence on MT stability; however, MAPs isolated from nerves did not show any prominent maturational change. Posttranslational modifications of MAPs that do not change their electrophoretic mobilities would not have been detected with the current method and remain as hypothetical mechanisms for maturational increases in stability. The technique employed may also isolate some subset of the total MAPs in the nerves. In particular, the MAPs associated with labile MTs are likely to be overrepresented, because the MAP preparation depends on solubilizing the MTs and their MAPs at the initial step. The use of a freeze/ thaw cycle before homogenization was intended to partially disrupt the stable MTs and release their MAPs; however, the exact contribution of stable and labile MTs to the MAP preparations remains unknown. The "microtubule-associated" proteins in peripheral nerves, operationally defined, include MAPs different from the brain. Localization of these proteins in situ and definition of their relationship to established MAPs remain to be accomplished.

\section{Adaptive significance of stable $M T$}

Mature axons contain a large population of highly stable MT (Brady et al., 1984; Donoso, 1986; Sahenk and Brady, 1987; Filliatreau et al., 1988) as compared to other cells (Behnke and Forer, 1967; Pipeleers et al., 1977b; Schulze and Kirschner, 
1987; Sammak and Borisy, 1988). The function of these stable MTs is not clearly understood, but some hypotheses merit consideration. The axon must remain as an extended cell process throughout the life of an animal, and stable MTs that are stationary or moving very slowly may serve to stabilize axonal structure. Stable MTs might also be important mechanical substrates for fast axonal transport. The distal axon remains critically dependent on a continuing supply of materials via fast axonal transport, and it is likely that the cell body requires uninterrupted retrograde transport to maintain normal function. Indeed, a number of drugs that destabilize MTs are known to interfere with fast axonal transport: colchicine (Kreutzberg, 1969), vincristine (Green et al., 1977), maytansine (Donoso et al., 1978), and nocodazole (Samson et al., 1979). One possible basis for the disruption of axonal transport by agents that alter MT stability has been suggested by recent work showing that 2 of the intracellular "motors" that are likely to drive axonal transport depend on a physical interaction with assembled MTs: kinesin (Vale et al., 1985a, b) and MAP1C (Paschal and Vallee, 1987; Paschal et al., 1987). Hence, the presence of a large population of highly stabilized axonal MTs may be a safeguard to ensure uninterrupted anterograde and retrograde fast axonal transport.

An alternative view of the increase in MT stability with axonal maturation is that immature axons must transport a larger proportion of tubulin in the form of subunits or readily depolymerizable MTs in order to support distal axonal elongation (Bamburg et al., 1986) or other developmental changes that require a high degree of plasticity of the axonal cytoskeleton. In this case, the later accumulation of stable MTs that move slowly (or do not move) may reflect an economy of metabolic effort in mature axons, which no longer require such an abundance of tubulin subunits to reach their distal regions.

The present observations give little insight into the physical form of the tubulin undergoing slow axonal transport. A measurable amount of transported tubulin was associated with all 3 fractions, unassembled subunits, labile MTs and stable MTs for all the conditions studied. The time resolution of such experiments is not sufficient to determine which forms of tubulin are undergoing transport at any instant, because interconversion between subunits and labile MTs could be much more rapid than axonal transport over the macroscopic distances studied. Clarification of the relationship of the highly stabilizd form of MTs in axons to the varied biologic functions of MTs poses a major challenge for future investigation.

\section{References}

Bamburg JR, Bray D, Chapman K (1986) Assembly of microtubules at the tip of growing axons. Nature 321:788-790.

Baudier J, Cole RD (1988) Interactions between the microtubuleassociated y proteins and $S 100 \mathrm{~b}$ regulate y phosphorylation by the $\mathrm{Ca}^{2+} /$ calmodulin-dependent protein kinase II. J Biol Chem 263:58765883.

Behnke P, Forer A (1967) Evidence for four classes of microtubules in individual cells. J Cell Sci 2:169-192.

Binet S, Meininger V (1988) Biochemical basis of microtubule cold stability in the peripheral and central nervous systems. Brain Res 450: 231-236.

Black MM, Keyser P (1987) Acetylation of alpha-tubulin in cultured neurons and the induction of alpha-tubulin acetylation in PC-12 cells by treatment with nerve growth factor. J Neurosci 7:1833-1842.

Black MM, Baas PW, Humphries S (1989) Dynamics of alpha-tubulin deacetylation in intact neurons. J Neurosci 9:358-368.

Bradley WG, Lassman LP, Pearce GW (1970) The neuromyopathy of vincristine in man: clinical, electrophysiological and pathological studies. J Neurol Sci 10:107-131.
Brady ST, Tytell M, Lasek RJ (1984) Axonal tubulin and axonal microtubules: biochemical evidence for cold stability. J Cell Biol 99: 1716-1724.

Bulinski JC, Richards JE, Piperno G (1988) Posttranslational modifications of alpha tubulin: detyrosination and acetylation differentiate populations of interphase microtubules in cultured cells. J Cell Biol 106:1213-1220.

Cambray-Deakin M, Burgoyne RD (1987) Acetylated and detyrosinated alpha-tubulins are co-localized in stable microtubules in rat meningeal fibroblasts. Cell Motil Cytoskel 8:284-291.

Denoulet P, Filliatreau G, de Neuchaud B, Di Giamberardino L (1989) Differential axonal transport of isotubulins in the motor axons of the rat sciatic nerve. J Cell Biol 108:965-971.

Detrich HW III, Prasad V, Luduena RF (1987) Cold-stable microtubules from antarctic fishes contain unique alpha tubulins. J Biol Chem 262:8360-8366.

Donoso JA (1986) Microtubule stability along mammalian peripheral nerves. J Neurobiol 17:383-403.

Donoso JA, Watson DF, Heller-Bettinger IE, Samson FE (1978) Maytansine action on fast axoplasmic transport and ultrastructure of vagal axons. Cancer Res 38:1633-1637.

Faivre C, Legrand C, Rabie A (1985) The microtubular apparatus of cerebellar Purkinje cells during postnatal development of the rat: the density and cold-stability of microtubules increase with age and are sensitive to thyroid hormone. Int J Dev Neurosci 3:559-565.

Filliatreau G, Denoulet P, de Neuchaud B, Di Giamberardino L (1988) Stable and metastable cytoskeltal polymers carried by slow axonal transport. J Neurosci 8:2227-2233.

Gottschalk PG, Dyck PJ, Kiely JM (1968) Vinca alkaloid neuropathy: nerve biopsy studies in rats and in man. Neurology 18:875-882.

Green LS, Donoso JA, Heller-Bettinger IE, Samson FE (1977) Axonal transport disturbances in vincristine-induced peripheral neuropathy. Ann Neurol 1:255-262.

Gundersen GG, Khawaja S, Bulinski JC (1987) Postpolymerization detyrosination of alpha-tubulin: a mechanism for subccllular differentiation of microtubules. J Cell Biol 105:251-264.

Hesketh JE (1984) Differences in polypeptide composition and enzyme activity between cold-stable and cold-labile microtubules and study of microtubule alkaline phosphatase activity. FEBS Lett 169: 313-318.

Hoffman PN, Cleveland DW (1988) Neurofilament and tubulin expression recapitulates the developmental program during axonal regeneration: induction of a specific beta tubulin isotype. Proc Natl Acad Sci USA 85:4530-4533.

Hoffman PN, Lasek RJ, Griffin JW, Price DL (1983) Slowing of the axonal transport of neurofilament proteins during development. $\mathrm{J}$ Neurosci 3:1694-1700.

Hoffman PN, Griffin JW, Cold BG, Price DL (1985) Slowing of neurofilament transport and the radial growth of developing nerve fibers. J Neurosci 5:2920-2929.

Hoshi M, Akiyama T, Shinohara Y, Miyata Y, Ogawara H, Nishida E, Sakai H (1988) Protein-kinase-C-catalyzed phosphorylation of the microtubule-binding domain of microtubule-associated protein 2 inhibits its ability to induce tubulin polymerization. Eur $\mathbf{J}$ Biochem 174:225-230.

Job D, Rauch CT, Margolis RL (1987) High concentrations of STOP protein induce a microtubule super-stable state. Biochem Biophys Res Commun 148:429-435.

Komiya Y (1980) Slowing with age of the rate of slow axonal flow in bifurcating axons of rat dorsal root ganglion cells. Brain Res 183:477480.

Kreis TE (1987) Microtubules containing detyrosinated tubulin are less dynamic. EMBO J 6:2597-2606.

Kreutzberg GW (1969) Neural dynamics and axonal flow. VI. Blockage of intraaxonal enzyme transport by colchicine. Proc Natl Acad Sci USA 62:722-728.

Kuncl RW, Duncan G, Watson DF, Alderson K, Rogawski MA, Peper $M$ (1987) Colchicine myopathy and neuropathy. New Engl J Med 316:1562-1568.

Lim SS, Sammak PJ, Borisy GG (1989) Progressive and spatially differentiated stability of microtubules in developing neuronal cells. J Cell Biol 109:253-263.

McLeod JG, Penny R (1969) Vincristine neuropathy: an electrophysiological and histological study. J Neurol Neurosurg Psychiatry 32: 297-304.

Nixon RA, Logvinenko KB (1986) Multiple fates of newly synthesized 
neurofilament proteins: evidence for a stationary neurofilament network distributed nonuniformly along axons of retinal ganglion cell neurons. J Cell Biol 102:647-659.

Ohta K, Seo N, Yoshida T, Hiraga K, Tuboi S (1987) Tubulin and high molecular weight microtubule-associated proteins as endogenous substrates for protein carboxymethyltransferase in brain. Biochimie 69:1227-1234.

Olmsted JB (1981) Tubulin pools in differentiating neuroblastoma cells. J Cell Biol 89:418-423.

Paschal BM, Vallee RB (1987) Retrograde transport by the microtubule-associated protein MAP1C. Nature 330:181-183.

Paschal BM, King SM, Moss AG, Collins CA, Vallee RB, Witman GB (1987) Isolated flagellar outer arm dynein translocates brain microtubules in vitro. Nature 330:672-674.

Pipeleers DG, Pipeleers-Marichal MA, Sherline P, Kipnis DM (1977a) A sensitive method for measuring polymerized and depolymerized forms of tubulin in tissues. J Cell Biol 74:341-350.

Pipeleers DG, Pipeleers-Marichal MA, Kipnis DM (1977b) Physiological regulation of total tubulin and polymerized tubulin in tissues. J Cell Biol 74:351-357.

Piperno G, Fuller MT (1985) Monoclonal antibodies specific for an acetylated form of alpha-tubulin recognize the antigen in cilia and flagella from a variety of organisms. J Cell Biol 101:2085-2094.

Piperno G, LeDizet M, Chang X (1987) Microtubules containing acetylated alpha-tubulin in mammalian cells in culture. J Cell Biol 104: 289-302.

Riggs JE, Schochet SS, Gutmann L, Crosby TW, DiBartolomeo AG (1986) Chronic human colchicine neuropathy and myopathy. Arch Neurol 43:521-523.

Sahenk Z, Brady ST (1987) Axonal tubulin and microtubules: morphologic evidence for stable regions on axonal microtubules. Cell Motil Cytoskel 8:155-164.

Sale WS, Besharse JC, Piperno G (1988) Distribution of acetylated alpha-tubulin in retina and in in-vitro-assembled microtubules. Cell Motil Cytoskel 9:243-253.

Sammak PJ, Borisy GG (1988) Direct observation of microtubule dynamics in living cells. Nature 332:724-726.
Samson FE, Donoso JA, Heller-Bettinger IE, Watson DF, Hines RH (1979) Nocodazole action on tubulin assembly, axonal ultrastructure, and fast axoplasmic transport. J Pharmacol Exp Ther 208:411-417.

Schulze E, Kirschner M (1986) Microtubule dynamics in interphase cells. J Cell Biol 102:1020-1031.

Schulze E, Kirschner M (1987) Dynamic and stable populations of microtubules in cells. J Cell Biol 104:277-288.

Schulze E, Asai DJ, Bulinski JC, Kirschner M (1987) Posttranslational modification and microtubule stability. J Cell Biol 105:2167-2178.

Tashiro T, Komiya Y (1989) Stable and dynamic forms of cytoskeletal proteins in slow axonal transport. J Neurosci 9:760-768.

Tashiro T, Kurokawa M, Komiya Y (1984) Two populations of axonally transported tubulin differentiated by their interactions with neurofilaments. J Neurochem 43:1220-1225.

Towbin H, Staeblin T, Gordon J (1979) Electrophoretic transfer of proteins from polyacrylamide gels to nitrocellulose sheets: procedure and some applications. Proc Natl Acad Sci USA 76:4350-4354.

Tucker RP, Binder LI, Matus AI (1988) Neuronal microtubule-associated proteins in the embryonic avian spinal cord. J Comp Neurol 271:44-55.

Vale RD, Reese TS, Sheetz MP (1985a) Identification of a novel forcegenerating protein, kinesin, involved in microtubule-based motility. Cell 42:39-59.

Vale RD, Schnapp BJ, Mitchison T, Steuer E, Reese TS, Sheetz MP (1985b) Different axoplasmic proteins generate movement in opposite directions along microtubules in vitro. Cell 42:623-632.

Vallee R (1982) A taxol-dependent procedure for the isolation of microtubule-associated proteins (MAPs). J Cell Biol 92:435-442.

Watson DF, Hoffman PN, Fittro KP, Griffin JW (1989) Neurofilament and tubulin transport slows along the course of mature motor axons. Brain Res 477:225-232.

Yamamoto H, Fukunaga K, Goto S, Tanaka E, Miyamoto E (1985) $\mathrm{Ca}^{2+}$, calmodulin-dependent regulation of microtubule formation via phosphorylation of microtubule-associated protein 2 , tau factor, and tubulin, and comparison with the cyclic AMP-dependent phosphorylation. J Neurochem 44:759-768. 